\title{
Calculated model of wedge-shaped sliding supports taking into account rheological properties of viscoelastic lubricant
}

\author{
Akhverdiev Kamil Samedovich \\ «Rostov state transport university» (RSTU), \\ 2, Rostovskogo Strelkovogo Polka Narodnogo Opolcheniya \\ sq., Rostov-on-Don, 344038, Russia \\ vm_2@kaf.rgups.ru
}

\author{
Lagunova Elena Olegovna \\ «Rostov state transport university» (RSTU), \\ 2, Rostovskogo Strelkovogo Polka Narodnogo Opolcheniya \\ sq., Rostov-on-Don, 344038, Russia \\ lagunova@rambler.ru
}

\author{
Mukutadze Murman Alexsandrovich \\ «Rostov state transport university» (RSTU), \\ 2, Rostovskogo Strelkovogo Polka Narodnogo Opolcheniya \\ sq., Rostov-on-Don, 344038, Russia \\ murman1963@yandex.ru
}

\begin{abstract}
The paper presents a method for the formation of an exact self-similar solution of the hydrodynamic calculation of a wedge-shaped support (slider, guide) operating on viscoelastic liquid lubricant caused by the melt of the guide, taking into account the dependence of viscosity and shear modulus on temperature.
\end{abstract}

The solution of the problem has been found on the basis of a system of equations describing the motion of an incompressible liquid viscoelastic lubricant for the case of a "thin layer", taking into account the dependence of the viscosity of the liquid lubricant and the shear modulus on temperature, the continuity equation and the expression for the dissipation rate of mechanical energy for determining the function due to the melt of the guide surface coated with a melt of a low-melting coating. Asymptotic solution of a system of differential equations with allowance for the boundary conditions on the surface of the guide, slider and contour is found in the form of series in powers of the small parameter $K$, caused by the melt and the rate of dissipation of mechanical energy. In order to determine the velocity and pressure fields in the lubricating and molten layer, there is an exact self-similar solution for the zero and first approximations. As a result of finding the exact self-similar solution, the value of the function, caused by the melt of the guide (parameter M, characterizing the thickness of the molten film) has been found.

The influence of the parameter caused by the melt of the surface of the low-melting coating of the bearing bush on the bearing capacity and the friction force has been evaluated.

Keywords - wedge slide support (slider, guide); dependence of viscosity of lubricant on temperature; energy dissipation rate; molten guide surface; viscoelastic lubricant

\section{INTRODUCTION}

As you know, in the modern machine-building, tribo nodes for new machines are designed, as a rule, taking into account the increase in the static and shock loads acting on the sliding supports, which is determined by the tasks of modern engineering practice. It should be noted that one of the most important equitable components of fluid friction bearings is the lubricating medium.

When transmitting high powers by worm gearboxes of tribosystems using a mineral oil as a lubricant, difficulties occur due to the lack of lubricant necessary to prevent setting and raising the temperature of the working surfaces

In order to avoid such situations, as well as at high temperatures and stresses without any restrictions for sliding bearings, it is proposed to use a melt of low-melting metals as an additional lubricant.

At present, there are a large number of diffusion metallization technologies, with most of these technologies being complex, requiring the use of expensive equipment, which makes them industrially unrealizable. Against the background of these technologies, the technology of diffusion metallization of steels from low-melting liquid-metal solutions is favorably highlighted. The promising nature of this technology is related to the simplicity of its implementation, the possibility of using standard thermal equipment, combining this technology with the thermal treatment of coated products, etc., and, most importantly, with the possibility of obtaining quality products and regulating the properties of these coatings by varying the technology regimes. 
Diffusive metallization from low-melting liquid-metallic solutions is based on the phenomenon of selective isothermal transfer of metallic elements to metallic materials. Technologically, this process is performed by immersing and holding products in a low-melting melt containing an element in the dissolved state on the basis of which diffusion coatings are formed.

The use of this technology makes it possible to obtain single-component and multicomponent diffusion coatings on the surface of products based on various metallic elements.

Lubrication with a melt was studied in many applied problems, in particular, in the processes of forming and cutting metals [1-12]. A large number of works has been devoted to hydrodynamic calculation of sliding bearings in the absence of a lubricant and taking into account the dependence of the viscosity of the lubricant on pressure. A significant drawback of the friction pair operating on melt lubrication is a low loadbearing capacity. In addition, the lubrication process with the lubricant is not self-sustaining.

The works devoted to theoretical analysis of the work of radial and thrust sliding bearings in the presence of a lubricant of a tribological system caused by the melt of a surface covered with a low-melting metallic melt when taking into account the dependence of the viscosity of the lubricant on pressure [13-15].

A lot of works were dedicated to the development of a design model for radial and thrust sliding bearings, taking into account the rheological properties of a micropolar, viscoelastic lubricant, taking into account the dependence of the viscosity of the lubricant on the pressure and melt of the fusible coating. [16-23].

In this paper, the solution of the problem is given for the case when the lubricant has viscoelastic properties, taking into account the dependence of the viscosity of the lubricant and the shear modulus on temperature, as well as the melt of the low-melting coating.

\section{TASK SETTING}

The turbulent flow of a viscoelastic lubricant between an inclined slide and a guide is considered. It is assumed that the surfaces of the slider and the guide are separated by a layer of lubricant having viscoelastic properties, the slider is stationary, and the guide made of a material with a low melting point moves toward the narrowing of the gap at a speed $u^{*}$ (Fig. 1).

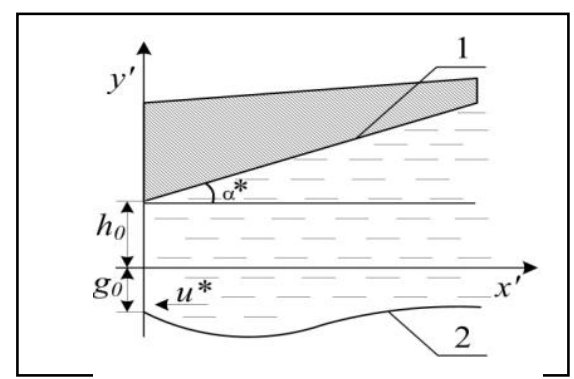

Fig. 1. Working scheme
In the Cartesian coordinate system $x^{\prime} O y^{\prime}$, the equation of the contour of the slider and the molten surface of the guide can be written in the form:

$$
y^{\prime}=h_{0}+x^{\prime} \operatorname{tg} \alpha^{*}, \quad y^{\prime}=-\Phi x^{\prime},
$$

where $\Phi x^{\prime}$ is thickness of the molten film in the initial section; $\alpha^{*}$ is the angle of slope of the linear contour of the slider to the axis $O x ; h_{0}$ is the thickness of the lubricating film in the initial section; $l$ is the length of the fixed working surface of the bearing (slider).

We assume that the dependence of the viscosity and shear modulus on pressure and temperature is expressed by formulas:

$$
\mu^{\prime}=\mu_{0} e^{-\alpha^{\prime} T^{\prime}-\tilde{T}_{0}}, G^{\prime}=G_{0} e^{-\alpha^{\prime} T^{\prime}-\tilde{T}_{0}},
$$

where $\mu^{\prime}$ is coefficient of dynamic viscosity of the lubricant; $\mu_{0}$ is the characteristic viscosity of a Newtonian lubricant; $\sigma^{\prime}$ is the experimental constant; $T^{\prime}=T \quad x^{\prime}$ is the temperature in the lubricating layer, $\tilde{T}_{0}$ is the initial temperature; $G_{0}$ is the characteristic value of the shear modulus, $G^{\prime}$ is the shear modulus.

When forming the analytical solution of the problem under consideration, the following assumptions are made:

1. Pressure $p$ is constant on the thickness of the lubricating film, given by the equations (1).

2. The liquid medium is a viscous incompressible fluid.

3. All the heat released in the lubricating film goes to the melting surface of the material of the guide.

4. The effect of turbulence can be reflected with the aid of the coefficient $j>1$, to which the viscosity should be multiplied in order to obtain the effective viscosity value. In addition, it is assumed that this coefficient $j$ can be expressed as the following function of the Reynolds number $j=0,0139 \mathrm{Re}^{0,657}$, where $\operatorname{Re}=\mathrm{c} u^{*} h_{0}^{2} / \mathrm{M}_{0} l$ is the Reynolds number, $\mathrm{c}$ is density, $u^{*}$ is movement rate slider, $l$ is the length of the fixed working surface of the bearing (slider).

\section{INITIAL EQUATIONS AND BOUNDARY CONDITIONS}

As the initial equations, we consider a system of dimensionless equations of motion of a lubricant having viscoelastic properties for the case of a "thin layer", the 
continuity equation, and the energy dissipation rate formula, for determining the function $\Phi x$, caused by the molten guide:

$$
\begin{gathered}
\frac{\partial^{2} v}{\partial y^{2}}=\frac{1}{j \mu x} \frac{d p}{d x}+\frac{\beta}{j \mu x} \frac{d^{2} p}{d x^{2}}, \quad \frac{\partial u}{\partial y}+\frac{\partial v}{\partial x}=0, \\
-\frac{1}{j \mu x} \frac{d \Phi x}{d x}=-K \int_{-\Phi x}^{h x}\left(\frac{\partial v}{\partial y}\right)^{2} d y,
\end{gathered}
$$

here $u, v$ are components of the velocity vector of the lubricating medium; $p$ is hydrodynamic pressure in the lubricating layer; $\mu$ is coefficient of dynamic viscosity, $\beta=\frac{\mu_{0} u^{*}}{G l h}$ is Deborah number; $K=\frac{2 \mu_{0} u^{*} l}{h_{0}^{2} L}$ is the parameter characterizing the rate of dissipation of mechanical energy; $L$ is the specific heat of fusion per unit volume.

The system of equations (3) is solved using the following boundary conditions:

$$
\begin{gathered}
u=0, \quad v=0 \quad \text { at } y=1+\eta x=h x, \\
u=0, \quad v=-1 \quad \text { at } y=-\Phi x, \\
p 0=p 1=\frac{p_{a}}{p^{*}},
\end{gathered}
$$

where $\eta=\frac{\operatorname{ltg} \alpha^{*}}{h_{0}}$.

Assuming that the lubricant enters the bearing in the absence of an elastic deformation component, the characteristic of the special state of the lubricating medium will be written as follows:

$$
\begin{gathered}
\frac{d^{2} p}{d x^{2}}=0 \quad \text { at } x=0 ; \\
\Phi x=\tilde{g}_{0}=K g_{0}=0 \text { at } x=0 .
\end{gathered}
$$

The relations between dimensionless and dimensional quantities are given in the form:

$$
\begin{gathered}
u^{\prime}=u^{*} u, \quad v^{\prime}=u^{*} \varepsilon v, \quad x^{\prime}=l x, \quad y^{\prime}=h_{0} y, \\
p^{\prime}=p^{*} p, \quad T^{\prime}=T^{*} T, \quad \mathrm{e}=\frac{h_{0}}{l}, \quad \mathrm{M}^{\prime}=\mathrm{M}_{0} \mathrm{M}, \quad G^{\prime}=G_{0} G, \\
\sigma=\sigma^{\prime} T^{*}, \quad p^{*}=\frac{\mathrm{M}_{0} u^{*} l}{h_{0}^{2}} .
\end{gathered}
$$

Taking $K$, caused by the melt and the energy dissipation rate as a small parameter, we seek the function $\Phi{ }^{-}$as:

$$
\Phi x=-K \Phi_{1} x-K^{2} \Phi_{2} x-K^{3} \Phi_{3} x-\ldots=H .
$$

Boundary conditions for the dimensionless velocity components $u$ and $v$ on the contour $y=0-\Phi x$ can be written as:

$$
\begin{aligned}
& v 0-H x=v 0-\left.\left(\frac{\partial v}{\partial y}\right)\right|_{y=0} H x-\left.\left(\frac{\partial^{2} v}{\partial y^{2}}\right)\right|_{y=0} H^{2} x-\ldots=-1 ; \\
& u 0-H x=u 0-\left.\left(\frac{\partial u}{\partial y}\right)\right|_{y=0} H x-\left.\left(\frac{\partial^{2} u}{\partial y^{2}}\right)\right|_{y=0} H^{2} x-\ldots=0 .
\end{aligned}
$$

We seek the asymptotic solution of the system of differential equations (3) with allowance for the boundary conditions (4) in the form of series in powers of the small parameter $K$ :

$$
\begin{gathered}
v=v_{0}(x, y)+K v_{1}(x, y)+K^{2} v_{2}(x, y)+\ldots, \\
u=u_{0}(x, y)+K u_{1}(x, y)+K^{2} u_{2}(x, y)+\ldots, \\
\Phi(x)=-K \Phi_{1}(x)-K^{2} \Phi_{2}(x)-K^{3} \Phi_{3}(x)-\ldots, \\
T=T_{0}(x)+K T_{1}(x)+K^{2} T_{2}(x)+K^{3} T_{3}(x) \ldots, \\
\mathrm{M}=\mathrm{M}_{0}(x)+K \mathrm{M}_{1}(x)+K^{2} \mathrm{M}_{2}(x)+K^{3} \mathrm{M}_{3}(x) \ldots
\end{gathered}
$$

Adding (8) to the system of differential equations (3) taking into account the boundary conditions (4), we obtain the following equations:

- for the zero approximation:

$$
\frac{\partial^{2} v_{0}}{\partial y^{2}}=\frac{1}{\mathrm{jM}_{0} x} \frac{d p_{0}}{d x}+\frac{\text { в }}{\mathrm{jM}_{0} x} \frac{d^{2} p_{0}}{d x^{2}}, \frac{\partial v_{0}}{\partial x}+\frac{\partial u_{0}}{\partial y}=0
$$

With boundary conditions:

$$
\begin{gathered}
v_{0}=0, \quad u_{0}=0, \quad \text { at } y=1+\eta x, \\
v_{0}=-1, \quad u_{0}=0 \text { at } y=0, \\
p_{0}^{\prime \prime} 0=0 ; \quad p_{0} \quad 0=p_{0} \quad 1=\frac{p_{a}}{p^{*}} ; \\
K \Phi_{0} \quad 0=K g_{0}=0 ;
\end{gathered}
$$


- for the first approximation up to the members $O\left(\frac{K}{\mathrm{M}_{0}^{2}}\right)$ :

$$
\begin{gathered}
\frac{\partial^{2} v_{1}}{\partial y^{2}}=\frac{1}{\mathrm{jm}_{0} x}\left(\frac{d p_{1}}{d x}+\beta \frac{d^{2} p_{1}}{d x^{2}}\right), \frac{\partial v_{1}}{\partial x}+\frac{\partial u_{1}}{\partial y}=0, \\
\frac{1}{\mathrm{jm}_{0} x} \frac{d \Phi_{1}(x)}{d x}=\int_{0}^{1+\eta x}\left(\frac{\partial v_{0}}{\partial y}\right)^{2} d y,
\end{gathered}
$$

with boundary conditions:

$$
\begin{gathered}
v_{1}=\left.\left(\frac{\partial v_{0}}{\partial y}\right)\right|_{y=0} \cdot \Phi_{1} x, u_{1}=\left.\left(\frac{\partial u_{0}}{\partial y}\right)\right|_{y=0} \cdot \Phi_{1} x, \\
v_{1}=0, \quad u_{1}=0 \quad \text { at } \quad h x=1+\eta x, \\
\mathrm{C}_{0}^{\prime} 0=0 ; \quad p_{1}^{\prime \prime} 0=0, \quad p_{1} 0=p_{1} 1=0, \\
K \Phi_{1} 0=K \tilde{\tilde{\alpha}}^{*}, \quad \Phi 0=\Phi 1=\tilde{\tilde{\alpha}}^{*} .
\end{gathered}
$$

The exact solution of the problem for the zero approximation will be sought by:

$$
\begin{gathered}
u_{0}=-\frac{\partial \psi_{0}}{\partial x}+U_{0} x, y, \quad v_{0}=\frac{\partial \psi_{0}}{\partial y}+V_{0} x, y, \\
\psi_{0} x, y=\tilde{\psi}_{0} \xi, \quad \xi=\frac{y}{h x}, \\
V_{0} x, y=\tilde{v} \xi, \quad U_{0} x, y=-\tilde{u}_{0} \xi \cdot h^{\prime} x .
\end{gathered}
$$

Adding (13) to the system of differential equations (9), taking into account the boundary conditions (10), we obtain the following system of differential equations:

$$
\begin{gathered}
\tilde{\psi}_{0}^{\prime \prime \prime}=\tilde{C}_{2}, \quad \tilde{v}_{0}^{\prime \prime}=\tilde{C}_{1}, \quad \tilde{u}_{0}^{\prime}+\xi \tilde{v}_{0}^{\prime}=0, \\
\frac{\beta}{\mathrm{jM}_{0} x} \frac{d^{2} p_{0}}{d x^{2}}+\frac{1}{\mathrm{jM}_{0} x} \frac{d p_{0}}{d x}=\frac{\tilde{C}_{1}}{h^{2} x}+\frac{\tilde{C}_{2}}{h^{3} x}
\end{gathered}
$$

And boundary conditions:

$$
\tilde{\Psi}_{0}^{\prime} \quad 0=0, \quad \tilde{\psi}_{0}^{\prime} 1=0, \quad \tilde{u}_{0} 1=0, \quad \tilde{v}_{0} 1=0,
$$

$\tilde{u}_{0} 0=0, \quad \tilde{v}_{0} 0=-1, \int_{0}^{1} \tilde{v}_{0} \xi d \xi=0, \quad p_{0} 0=p_{0} \quad 1=\frac{p_{a}}{p^{*}}$.

By the direct integration we obtain:

$$
\begin{aligned}
\tilde{\psi}_{0}^{\prime} \xi=\frac{\tilde{C}_{2}}{2} \xi^{2}-\xi, \tilde{v}_{0} \mathrm{o} & =\tilde{C}_{1} \frac{\mathrm{o}^{2}}{2}-\left(1+\frac{\tilde{C}_{1}}{2}\right) \mathrm{o}+1, \\
\tilde{\mathrm{C}}_{1} & =6 .
\end{aligned}
$$

From the condition $p_{0} 0=p_{0} 1=\frac{p_{a}}{p^{*}}$ and $p_{0}^{\prime \prime} 0=0$ solving the equation $\frac{\beta}{\mathrm{jM}_{0} x} \frac{d^{2} p_{0}}{d x^{2}}+\frac{1}{\mathrm{jm}_{0} x} \frac{d p_{0}}{d x}=\frac{\tilde{C}_{1}}{h^{2} x}+\frac{\tilde{C}_{2}}{h^{3} x} \quad$ for $\quad \tilde{C}_{2} \quad$ we obtain:

$$
\tilde{C}_{2}=\frac{6 \eta-12 \eta \beta-6-12 \eta \beta^{2}\left(e^{-\frac{1}{\beta}}-1\right)}{1+3 \eta \beta^{2}-\frac{3}{2} \eta-3 \eta \beta^{2}\left(e^{-\frac{1}{\beta}}-1\right)}
$$

\section{Definition of Hydrodynamic PRESSURE}

The hydrodynamic pressure in the lubricating layer is determined from the equation $\frac{\beta}{\mathrm{jM}_{0} x} \frac{d^{2} p_{0}}{d x^{2}}+\frac{1}{\mathrm{jm}_{0} x} \frac{d p_{0}}{d x}=\frac{\tilde{C}_{1}}{h^{2} x}+\frac{\tilde{C}_{2}}{h^{3} x}$, first we need to find $\mathrm{m}_{0} x$. Having differentiated $\mathrm{m}_{0} \quad x=e^{-\alpha\left(T-\frac{\tilde{T}_{0}}{T^{*}}\right)}$ we obtain:

$$
\frac{d \mu_{0} x}{d x}=\mu_{0} x\left(-\alpha \frac{d T_{0}}{d x}\right)
$$

In order to $\frac{d T_{0}}{d x}$ we use the formula for the energy dissipation rate:

$$
\frac{d T_{0}}{d x}=-\frac{24 \mu_{0} \mu_{0} x u^{*} l h x}{T^{*} c_{p} h_{0}^{2} \tilde{C}_{2}} \int_{0}^{1}\left(\frac{\tilde{\psi}_{0}^{\prime \prime} \xi}{h^{2} x}+\frac{\tilde{v}_{0}^{\prime} \xi}{h x}\right)^{2} d \xi .
$$

Adding (19) to (18) and making a series of transformations, we obtain:

$$
\frac{1}{\mu_{0}^{2} x} \frac{d \mu_{0} x}{d x}=\frac{24 \mu_{0} u^{*} l \alpha h x}{T^{*} c_{p} h_{0}^{2} \tilde{C}_{2}} \int_{0}^{1}\left(\frac{\tilde{\psi}_{0}^{\prime \prime} \xi}{h^{2} x}+\frac{\tilde{v}_{0}^{\prime} \xi}{h x}\right)^{2} d \xi,
$$

where $c_{p}$ is the heat capacity at the constant pressure.

Integrating (20), we obtain: 


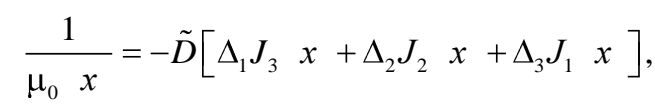

where $\tilde{D}=\frac{24 \mu_{0} \alpha u^{*} l}{T^{*} c_{p} h_{0}^{2} \tilde{C}_{2}} ; \quad \Delta_{1}=\int_{0}^{1} \tilde{\psi}_{0}^{\prime \prime} \xi^{2} d \xi=\frac{\tilde{C}_{2}^{2}}{12}$;

$$
\begin{gathered}
\Delta_{2}=2 \int_{0}^{1} \tilde{\psi}_{0}^{\prime \prime} \xi \cdot \tilde{v}_{0}^{\prime} \xi d \xi=\frac{1}{6} \tilde{C}_{1} \tilde{C}_{2} ; \\
\Delta_{3}=\int_{0}^{1} \tilde{v}_{0}^{\prime} \xi^{2} d \xi=4 ; \quad J_{k} \quad x=\int_{0}^{x} \frac{d x}{h^{k} x} .
\end{gathered}
$$

Substitute the function $\mu_{0} x$ by its averaged integral value:

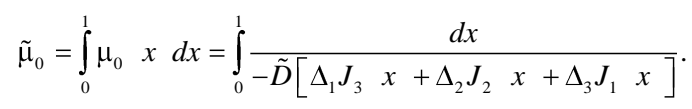

Solving the obtained equations $\Delta_{1}, \Delta_{2}$, $\Delta_{3}, J_{3} x, J_{2} x, J_{1} x$ within the accuracy to $O 3^{2}$ for $\tilde{\mu}$ we obtain the following expression:

$$
\tilde{\mu}_{0}=1+D \alpha\left(\frac{235}{8} \eta \beta^{2}\left(e^{-\frac{1}{\beta}}-1\right)+\frac{631}{24} \eta+\frac{49}{4}-\frac{47}{4} \eta \beta\right),
$$

where $D=\frac{24 \mu_{0} u^{*} l}{T^{*} c_{p} h_{0}^{2}}$

Then:

$$
\begin{aligned}
& p_{0}=j \tilde{\mu}_{0}\left[-15 \eta \beta^{2}+\eta \beta^{2} e^{-\frac{x}{\beta}}+6\left(x+2 \eta\left(\beta-\frac{x^{2}}{2}\right)\right)+\right. \\
& \left.+15 \eta x-6 x+6 \eta \beta x-15 \eta \beta^{2} x\left(e^{-\frac{1}{\beta}}-1\right)-18 \eta\left(\beta x-\frac{x^{2}}{2}\right)\right]+\frac{p_{a}}{p^{*}} .
\end{aligned}
$$

In order to define $\Phi_{1} x$ taking into account the equation (16), we arrive at the following equation:

$$
\frac{d \Phi_{1}}{d x}=j \tilde{\mu}_{0} h x \int_{0}^{1}\left(\frac{\psi_{0}^{\prime \prime} \xi}{h^{2} x}+\frac{\tilde{v}_{0}^{\prime} \xi}{h x}\right)^{2} d \xi .
$$

Integrating the equation (25), we get:

$$
\Phi_{1} x=j \tilde{\mathrm{M}}_{0}\left(\int_{0}^{x} \frac{\Delta_{1} d x}{h^{3} x}+\int_{0}^{x} \frac{\Delta_{2} d x}{h^{2} x}+\int_{0}^{x} \frac{\Delta_{3} d x}{h x}\right)
$$

Solving the equation account $K \Phi_{1} \quad 0=K \tilde{\tilde{\alpha}}^{*}$, we obtain:

taking into

The exact solution for the first approximation will be sought in the form:

$$
\begin{gathered}
u_{1}=-\frac{\partial \psi_{1}}{\partial x}+U_{1} x, y, \quad v_{1}=\frac{\partial \psi_{1}}{\partial y}+V_{1} x, y, \\
\psi_{1} x, y=\tilde{\psi}_{1} \xi, \quad \xi=\frac{y}{h x}, \\
V_{1} x, y=\tilde{v} \xi, \quad U_{1} x, y=-\tilde{u}_{1} \xi \cdot h^{\prime} x .
\end{gathered}
$$

Adding (28) to the system of differential equations (11), taking into account the boundary conditions (12), we obtain the following system of differential equations:

$$
\begin{gathered}
\tilde{\psi}_{1}^{\prime \prime \prime} \xi=\tilde{\tilde{C}}_{2}, \quad \tilde{v}_{1}^{\prime \prime}=\tilde{\tilde{C}}_{1}, \quad \tilde{u}_{1}^{\prime}+\xi \tilde{v}_{1}^{\prime}=0, \\
\frac{\beta}{j \mu_{1} x} \frac{d^{2} p_{1}}{d x^{2}}+\frac{1}{j \mu_{1} x} \frac{d p_{1}}{d x}=\frac{\tilde{\tilde{C}}_{1}}{h^{2} x}+\frac{\tilde{\tilde{C}}_{2}}{h^{3} x},
\end{gathered}
$$

And boundary conditions:

$\tilde{\psi}_{1}^{\prime} 0=0, \quad \tilde{\psi}_{1}^{\prime} 1=0, \quad \tilde{u}_{1} 1=0, \quad \tilde{v}_{1} 1=0$,

$\tilde{v}_{1} 0=M, \tilde{u}_{1} 0=0, \int_{0}^{1} \tilde{v}_{1} \xi d \xi=0, p_{1} 0=p_{1} 1=0$

By the direct integration we obtain:

$$
\begin{gathered}
\tilde{\psi}_{1}^{\prime} \xi=\frac{\tilde{\tilde{C}}_{2}}{2} \xi^{2}-\xi, \tilde{v}_{1} \mathrm{o}=\tilde{\tilde{C}}_{1} \frac{\mathrm{o}^{2}}{2}-\left(\frac{\tilde{\tilde{C}}_{1}}{2}+M\right) \mathrm{o}+M, \\
\tilde{\tilde{C}}_{1}=6 M
\end{gathered}
$$

where

$$
M=\left.\sup _{x \in 0 ; 1}\left(\frac{\partial v_{0}}{\partial y}\right)\right|_{y=0} \cdot \Phi_{1} \quad x=
$$




$$
\begin{aligned}
& =\sup _{x \in 0 ; 1}\left[1-3 x+63 x-\frac{15}{2} 3-63 \mathrm{~B}+\frac{15}{2} 3 \mathrm{~B}^{2}\left(e^{-\frac{1}{\mathrm{~B}}}-1\right)+93 \mathrm{в}-x\right] \times \\
& \times \mathrm{ju}_{0}\left[\left(x-\frac{3}{2} 3 x^{2}\right) \frac{\tilde{C}_{2}^{2}}{12}+\tilde{C}_{2} x-3 x^{2}+4\left(x-\frac{1}{2} 3 x^{2}\right)+6\right] .
\end{aligned}
$$

From the condition $p_{1} 0=p_{1} \quad 1=0$ for $\tilde{\tilde{C}}_{2}$ we obtain:

$$
\tilde{\tilde{C}}_{2}=M 15 \eta-6+6 \eta \beta-15 \eta \beta^{2}\left(e^{-\frac{1}{\beta}}-1\right)
$$

In order to determine the values $\tilde{\tilde{C}}_{2}$ and hydrodynamic pressure $p_{1}$ it is first necessary to find $\mu_{1} x$.Having differentiated $\mu_{1} x=e^{-\alpha\left(T_{1}-\frac{\tilde{T}_{0}}{T^{*}}\right)}$ as per $x$, we obtain:

$$
\frac{d \mu_{1} x}{d x}=-\alpha\left(\mu_{0} x \frac{d T_{1}}{d x}+\mu_{1} x \frac{d T_{0}}{d x}\right) .
$$

In order to define $\frac{d T_{1}}{d x}$ we use the formula for energy dissipation rate:

$$
\frac{d T_{1}}{d x}=-\frac{48 \mu_{0} \mu_{1} x u^{*} l h x}{T^{*} c_{p} h_{0}^{2} \tilde{\tilde{C}}_{2}} \int_{0}^{1}\left(\frac{\tilde{\Psi}_{0}^{\prime \prime} \xi}{h^{2} x}+\frac{\tilde{v}_{0}^{\prime} \xi}{h x}\right)\left(\frac{\tilde{\Psi}_{1}^{\prime \prime} \xi}{h^{2} x}+\frac{\tilde{v}_{1}^{\prime} \xi}{h x}\right) d \xi .
$$

Adding (19) and (34) to (33) and making a series of transformations, we obtain:

$\frac{1}{\tilde{\mu}_{0} \mu_{1} x} \frac{d \mu_{1} x}{d x}=\frac{48 \mu_{0} u^{*} l \alpha}{T^{*} c_{p} h_{0}^{2} \tilde{\tilde{C}}_{2}}\left(\frac{\tilde{\Delta}_{1}}{h^{3} x}+\frac{\tilde{\Delta}_{2}}{h^{2} x}+\frac{\tilde{\Delta}_{3}}{h^{2} x}+\frac{\tilde{\Delta}_{4}}{h x}\right)+$

$+\frac{24 \mu_{0} u^{*} l \alpha}{T^{*} c_{p} h_{0}^{2} \tilde{C}_{2}}\left(\frac{\Delta_{1}}{h^{3} x}+\frac{\Delta_{2}}{h^{2} x}+\frac{\Delta_{3}}{h x}\right)$,

where $\tilde{\Delta}_{1}=\int_{0}^{1} \tilde{\psi}_{0}^{\prime \prime} \xi \tilde{\Psi}_{1}^{\prime \prime} \xi d \xi ; \quad \tilde{\Delta}_{2}=\int_{0}^{1} \tilde{\psi}_{0}^{\prime \prime} \xi \cdot \tilde{v}_{1}^{\prime} \xi d \xi$;

$$
\tilde{\Delta}_{3}=\int_{0}^{1} \tilde{\psi}_{1}^{\prime \prime} \xi \cdot \tilde{v}_{0}^{\prime} \quad \xi d \xi ; \tilde{\Delta}_{4}=\int_{0}^{1} \tilde{v}_{0}^{\prime} \xi \cdot \tilde{v}_{1}^{\prime} \xi d \xi .
$$

Integrating (35) and replacing $\mu_{1} x$ by its averaged integral value, we obtain:

$$
\begin{aligned}
& \frac{1}{\tilde{\mu}_{0}} \ln \left|\tilde{\mu}_{1}\right|=\frac{24 \mu_{0} u^{*} l \alpha}{T^{*} c_{p} h_{0}^{2}}\left[\frac{\tilde{C}_{2}}{6}\left(\frac{1}{2}-\frac{\eta}{2}\right)+\frac{2 M \tilde{C}_{2}}{\tilde{C}_{2}}\left(\frac{1}{2}-\frac{\eta}{3}\right)-1+\right. \\
& \left.+\frac{2 \eta}{3}+\frac{8 M}{\tilde{\tilde{C}}_{2}}\left(\frac{1}{2}-\frac{\eta}{6}\right)+\left(\frac{235}{8} \eta \beta^{2}\left(e^{-\frac{1}{\beta}}-1\right)+\frac{631}{24} \eta+\frac{49}{4}-\frac{47}{4} \eta \beta\right)\right] .
\end{aligned}
$$

Solving the obtained equations $\tilde{\Delta}_{1}, \tilde{\Delta}_{2}, \tilde{\Delta}_{3}, \tilde{\Delta}_{4}$ within the accuracy to $O 3^{2}$ for $\tilde{\mu}_{1}$ we obtain:

$\tilde{\mu}_{1}=$

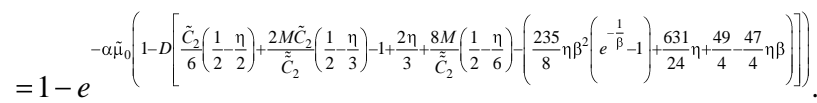

Then from the condition $p_{1} 0=p_{1} \quad 1=0$ and $p_{1}^{\prime \prime} 0=0$ for $p_{1}$ we obtain:

$$
\begin{aligned}
& p_{1}=j \tilde{\mu}_{1}\left[15 \eta \beta^{2}\left(e^{-\frac{x}{\beta}}-1\right) 1-x+6 M\left(x+23\left(\beta-\frac{x^{2}}{2}\right)\right)+\right. \\
& \left.+M\left(15 \eta x-6 x+6 \eta \beta x-183\left(\beta x-\frac{x^{2}}{2}\right)\right)\right],
\end{aligned}
$$

\section{RESUlTS OF RESEARCH AND THEIR DISCUSSION}

Let us now turn to the determination of the basic operating characteristics of the bearing. Taking into account (9), (11), (24) and (38) for the bearing capacity and the frictional force, we obtain:

$$
\begin{aligned}
& W=\frac{\mathrm{M}_{0} l^{2} u^{*}}{h_{0}^{2}} \int_{0}^{1}\left(p_{0}-\frac{p_{a}}{p^{*}}+K p_{1}\right) d x= \\
& =\frac{\mathrm{M}_{0} l^{2} u^{*} \mathrm{j}}{h_{0}^{2}}\left\{\tilde { \mathrm { M } } _ { 0 } j \left[-153 \mathrm{~B}^{2}-3 \mathrm{~B}\left(e^{-\frac{1}{\mathrm{~B}}}-1\right)+6\left(\frac{1}{2}+2 \mathrm{~B}\left(\mathrm{в}-\frac{1}{6}\right)\right)+\frac{15}{2} 3-3+33 \mathrm{~B}-\right.\right. \\
& \left.-\frac{15}{2} 3 \mathrm{~B}^{2}\left(e^{-\frac{1}{\mathrm{~B}}}-1\right)-183\left(\frac{\mathrm{B}}{2}-\frac{1}{6}\right)\right]+K \tilde{\mathrm{M}}_{1}\left[153 \mathrm{~B}\left(e^{-\frac{1}{\mathrm{~B}}}-2\right)-\right. \\
& -153 \mathrm{~B}^{2}\left(-\frac{1}{\mathrm{~B}} e^{-\frac{1}{\mathrm{~B}}}-\frac{1}{\mathrm{~B}^{2}}\left(e^{-\frac{1}{\mathrm{~B}}}-1\right)+\frac{1}{2}\right)+6 M\left(\frac{1}{2}+23\left(\mathrm{~B}-\frac{1}{6}\right)\right)+ \\
& \left.\left.+M\left(\frac{15}{2} 3-3+33 \mathrm{~B}-183\left(\frac{\mathrm{B}}{2}-\frac{1}{6}\right)\right)\right]\right\},
\end{aligned}
$$




$$
\begin{aligned}
& L_{m p}=\frac{\mathrm{M}_{0} l^{2} u^{*}}{h_{0}} \int_{0}^{1}\left[\left.\frac{\partial v_{0}}{\partial y}\right|_{y=0}+\left.K \frac{\partial v_{1}}{\partial y}\right|_{y=0}\right] d x= \\
& =\frac{\mathrm{M}_{0} l^{2} u^{*} j}{h_{0}}\left[\left(1-\frac{133}{2}+33 \text { в }\right) \tilde{\mathrm{M}}_{0}+\tilde{\mathrm{M}}_{1} K M+\right. \\
& \left.+\frac{15}{2} 3 \mathrm{~B}^{2}\left(e^{-\frac{1}{\mathrm{~B}}}-1\right) \tilde{\mathrm{M}}_{0}+\tilde{\mathrm{M}}_{1} K\right] .
\end{aligned}
$$

The input parameters for calculating the bearing capacity and the friction force, determined by expressions (39):

$$
\begin{gathered}
\mu_{0}=0.085 \mathrm{Hs} / \mathrm{m}^{2}, \eta=0.3 \ldots 1 \mathrm{~m}, l=0.1256 \ldots 0.1884 \mathrm{~m}, \\
u^{*}=1 . .3 \mathrm{~m} / \mathrm{s}, h_{0}=10^{-7} \ldots 2 \cdot 10^{-6} \mathrm{~m}, \text { щ } 0 \div 1 ; \mathrm{B}=0 \div 1 ; \\
K=0.0000022 \ldots 0.00052, M=6,47 \ldots 66,44,3=0,3 \div 1 ; \\
p_{a}=0.08 \div 0.101325 \mathrm{MПа}, \alpha=0 \ldots 1, L^{\prime}=35,33 \ldots 38,1 \mathrm{H} / \mathrm{m}^{2} .
\end{gathered}
$$

Based on the results of numerical analysis, the graphs are constructed (Figs 2-3), which allow us to draw the following conclusions.

1. A refined design model of a wedge-shaped sliding support operating under hydrodynamic lubrication conditions on a viscoelastic liquid lubricant caused by the surface melt of a low-melting surface of the guide surface.

2. The significant contribution of the parameters is shown: Deborah number $\mathrm{B}$, parameter $K$, caused by the surface melt of a low-melting surface of the guide surface, and parameter $\sigma$, characterizing the dependence of the viscosity of the lubricant on temperature.

3. It should be noted that when forming the contour of the slider profile parallel to the axis of abscissas, the loadbearing capacity of the thrust bearing intensively increases with increasing parameter $K$, in this case the bearing capacity is half that of the slider with the profile at an angle not equal to zero.

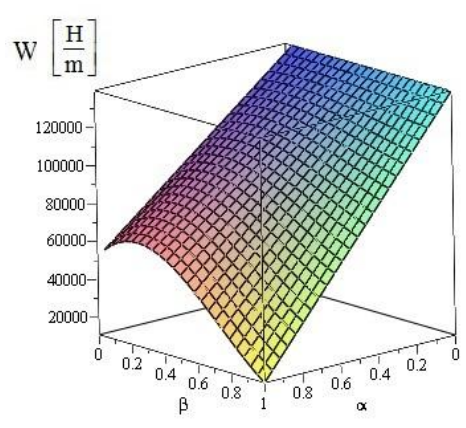

Fig. 2. Dependence of the bearing capacity on Deborah number в and on parameter $\alpha$, characterizing the dependence of the viscosity of the lubricant on temperature

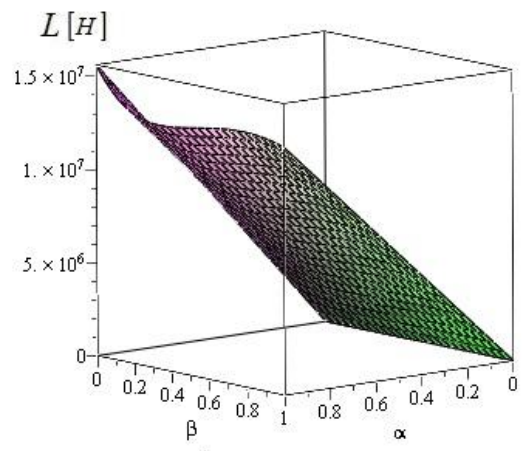

Fig. 3. Dependence of the friction force on Deborah number $\mathrm{B}$ and on parameter $\alpha$, characterizing the dependence of the viscosity of the lubricant on temperature

\section{Acknowledgment}

The work was has been made under the grant of Russian Railways No. 2210370/22.12.2016 for the development of scientific and pedagogical schools in the field of railway transport.

\section{References}

[1] D.Y. Kropachev, A.A. Grishin, A.D. Maslo, "Ways of Expeditious Measurement of Temperature of Fusion of Metals for Needs of Machine-Building Enterprises," Casting and Metallurgy, vol. 3(66), pp. 126-127, 2012.

[2] Wilson, Lubricant with Fusion" Problems of Friction and Lubricant, No. 1, pp. 19, 1976.

[3] Beretta, Niro, Silvestri, "The Sliding Bearings Greased by Own Fusion or a Product of Sublimation", The Works Amer. islands ing. - fur., No. 1, pp. 86-90, 1992.

[4] Physical Quantities. Reference book. Energoatomizdat, 1991.

[5] V.Y. Havin, Short Chemical Reference Book. L.: Chemistry, 1991.

[6] V.I. Perelman, Short Reference Book of the Chemist, M.-L.: Chemistry, 1964.

[7] Reference Book on Soldering. Prod. the 2nd, updated and revised. M.: Mechanical Engineering, 1984.

[8] L.I. Kotelnitskaya, N.N. Demidova, "Calculation Radial with Effective Work on Lubricant with Fusion in the Turbulent Mode", Vestnik RGUPS, No. 2, pp. 18-23, 2002.

[9] V.M. Prikhodko, L.I. Kotelnitskaya, "Mathematical Model of Hydrodynamic Lubricant When Melting a Basic Surface of the Radial Bearing”, Friction and Wear, vol. 22, No. 6, pp. 606-608, 2001.

[10] E.A. Zadorozhnaya, I.V. Mukhortov, I.G. Levanov, "Application of Non-Newtonian Models of Lubricant Fluids at Calculation ComplexLoaded Units of Friction Piston and Rotor-Type Machines", Friction and Lubricant in Machines And Mechanisms, No. 7, pp. 22 - 30, 2011.

[11] V.N. Prokopiev, A.K. Boyarshinova, E.A. Zadorojnaya, "Dynamics of the Complex-Loaded Bearing Greased with Non-Newtonian Liquid", Problem of Mechanical Engineering and Reliability of Machines, No. 6, pp. $108-114,2005$.

[12] V.N. Prokopiev, E.A. Zadorozhnaya, V.G. Karavaev, I.G. Levanov, "Improvement of the Computation Procedure for Complex-Loaded Sleeve Bearings Lubricated with Non-Newtonian Oils", Journal of Machinery Manufacture and Reliability, № 1, pp. 63-67, 2010.

[13] K.S. Akhverdiev, M.A. Mukutadze, E.O. Lagunova, K.S. Solop, "Working Out of an Analytical Model of a Radial Bearing Taking into Account Dependence of Viscous Characteristics of Micropolar Lubrication on Pressure and Temperature", International Journal of Applied Engineering Research, vol. 12, number 15 (2017), pp. 4840 4846, 2017. 
[14] E.O. Lagunova, "Simulation Model of Radial Bearing, Taking into Account the Dependence of Viscosity Characteristics of Micro-Polar Lubricant Material on Temperature", International Journal of Applied Engineering Research, vol. 12, number 12 (2017), pp. 3346-3352, 2017.

[15] E.O. Lagunova, "Design Model of Radial Bearing Taking into Account the Dependence of the Viscosity of Lubricant on Pressure and Temperature", Global Journal of Pure and Applied Mathematics, vol. 13, number 7 (2017), pp. 3531-3542, 2017.

[16] K.S. Akhverdiev, M.A. Mukutadze, A.C. Erkenov, Development of Systems of Settlement Models of Sliding Bearings on the Basis of Improvement of the Elastodynamic Theory Of Lubricant: Monograph Rostov n/D. RSTU, 371 pages, 2012.

[17] V.V. Vasilenko, E.O. Lagunova, M.A. Mukutadze, "Hydrodynamic Calculation of the Radial Bearing, the Lubricated Melt Coating in the Presence of a Lubricant”, Online journal «Naukovedenie», vol. 9, № 5, 2017. https://naukovedenie.ru/PDF/20TVN517.pdf

[18] K.S. Akhverdiev, E.O. Lagunova, V.V. Vasilenko, "Design Model of Radial Melt-Lubricated Bearing Taking into Account Pressure-Viscosity Ratio", Vestnik of Don State Technical University, vol. 17, no.3, pp. 2737, 2017.

[19] E.O. Lagunova, "Wedge-Shaped Sliding Supports Operating on Viscoelastic Lubricant Material Caused by the Melt, Taking Into
Account the Dependence of Viscosity and Shear Modulus on Pressure", International Journal of Applied Engineering Research, vol. 12, number 19, 2017, pp. 9120-9127, 2017.

[20] E.O. Lagunova, "Radial Plain Bearings Operating on Viscoelastic Lubricant Caused by the Melt, Taking into Account the Dependence of the Viscosity of the Lubricant and the Shear Modulus on the Pressure", International Journal of Applied Engineering Research, vol. 12, number 19, 2017, pp. 9128-9137, 2017.

[21] V.V. Vasilenko, E.O. Lagunova, M.A. Mukutadze, V.M. Prikhodko, "Calculation Model of the Radial Bearing, Caused by the Melt, Taking into Account the Dependence of Viscosity on Pressure", International Journal of Applied Engineering Research, vol. 12, number 19, 2017, pp. 9138-9148, 2017.

[22] K.S. Akhverdiev, M.A. Mukutadze, E.O. Lagunova, V.V. Vasilenko, "The Wedge-Shaped Sliding Support Using Micropolar Lubricant, Caused by Fusion", Vestnik RGUPS, No. 3 (67), pp. 8-15, 2017.

[23] E.O. Lagunova, M.A. Mukutadze, K.S. Solop, "Working Out of an Analytical Model of an Axial Bearing Taking into Account Dependence of Viscous Characteristics of Micropolar Lubrication on Pressure and Temperature", International Journal of Applied Engineering Research, vol. 12, number 14 (2017), pp. 4644-4650, 2017. 